\title{
A PHARMACOMETABONOMICS STUDY TO PREDICT AZATHIOPRINE (AZA)-INDUCED MYELOTOXICITY IN PATIENTS WITH RHEUMATOLOGICAL DISEASE
}

\author{
YAN-FANG LIN ${ }^{1 *}$, SONG-SEN SU ${ }^{2}$, HONG ZHOU ${ }^{1}$, LI-LI CAI ${ }^{1}$, and LEI HONG ${ }^{1}$ \\ ${ }^{1}$ Department of Pharmacy, \\ ${ }^{2}$ Department of Rheumatology, \\ Quanzhou First Hospital Affiliated to Fujian Medical University, \\ Quanzhou, Fujian 362000, China
}

\begin{abstract}
Azathioprine (AZA) is an immunosuppressive agent widely used in clinical therapy. However, the application of AZA is restricted by its myelotoxicity. AZA-induced myelotoxicity varies greatly among patients, so it is difficult to predict. In this study, untargeted metabolomics based on Ultra Performance Liquid Chromatography coupled with quadrupole time-of-flight mass spectrometry (UPLC Q-TOF/MS) was performed to identify metabolite biomarkers for predicting AZA-induced myelotoxicity in Chinese rheumatological disease patients. Fifty-two patients diagnosed with rheumatological disease at the Quanzhou First Hospital Affiliated with Fujian Medical University, Fujian, China, were enrolled in this study. Patients were assigned to an ADR group (patients with myelotoxicity) $(\mathrm{n}=15)$ or a NO ADR group (patients without myelotoxicity) $(n=15)$, and 22 patients without myelotoxicity withdrew, according to the presence of myelotoxicity. Serum was collected prior to treatment with AZA. Then, untargeted metabolomics based on LC/MS was performed to analyze the metabolic differences between the two groups. Several Kyoto Encyclopedia of Genes and Genomes (KEGG) pathways were significantly enriched in differential metabolites found between two groups $(\mathrm{P}<0.05$, impact $>0.1)$; the most enriched pathway, with an impact factor of 0.63 , was the Sphingolipid metabolism pathway. Twenty-two potential biomarkers were identified. Finally, two candidate biomarkers were selected to predict AZA-induced myelotoxicity, namely, sphingomyelin SM(d18:1/24:1(15Z)), phytosphingosine. They may be useful biomarkers for predicting AZA-induced myelotoxicity in Chinese patients with rheumatological disease. This study can aid future clinical studies of AZA-induced myelotoxicity and the development of personalized medicine.
\end{abstract}

Keywords: metabolomics, azathioprine, myelotoxicity, rheumatological disease

Rheumatic diseases are common in China (1). There are more than two hundred rheumatic diseases; some of the most common are rheumatoid arthritis (RA), ankylosing spondylitis (AS), systemic lupus erythematosus (SLE), and spondyloarthritis $(\mathrm{SpA})$. AZA is broadly applied in the treatment of rheumatic diseases $(2,3)$.

AZA is an immunosuppressant of purine analogues. It is metabolized to 6-mercaptopurine in vivo, which inhibits the synthesis of DNA and RNA and interferes with the function of CD4+T cells. AZA is very effective, especially in the treatment of SLE, but the incidence of adverse reactions is high. Common adverse reactions of AZA are blood system damage, liver function abnormality, infection, gastrointestinal reaction, respiratory system damage, and myelosuppression, among which myelosuppression is potentially lethal and warrants more attention in clinical practice $(4,5)$. Its incidence varies among situations and patients.

Previous studies have shown that thiopurine methyltransferase (TPMT) polymorphism leads to individual variation in 6-thioguanine nucleotides (6-TGNS) concentrations in patients after taking AZA. High levels of 6-TGNS increase the risk of myelotoxicity (6). For this reason, the Food and Drug Administration (FDA) recommends that patients be tested for TPMT genotype prior to treatment with AZA (7). However, recent studies have found that the adverse reactions of AZA cannot be fully explained by TPMT gene polymorphism. A study of 342 patients with SLE in Korea found no relationship between TPMT alleles and AZA toxicity (8). Therefore, it is difficult to ascertain based on TPMT

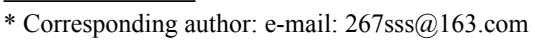


genotype test results alone whether AZA-induced myelotoxicity will occur. Recent evidence (9) indicates that NUDT15 c. $415 \mathrm{C}>\mathrm{T}$ is better than TPMT *3C at predicting AZA-induced myelotoxicity.

Metabonomics is a new technology that uses modern analytical techniques to measure dynamic changes in all endogenous metabolites (10). As a powerful analytical platform, metabonomics has many clinical applications, including the study of disease mechanisms and biomarkers, disease diagnosis, the impact of treatment on patients, drug metabolism, drug efficacy, and drug safety (11). The aim of pharmacometabonomics is to evaluate and predict the efficacy or toxicity of drugs by analyzing the metabolic phenotypes of organisms before and after administration (12). UPLC Q-TOF/MS is widely used because of its high resolution, analytic speed, and sensitivity $(13,14)$. Therefore, comprehensive analyses of the blood metabolism based on UPLC Q-TOF/MS are performed to identify differences between patients with and without hematological toxicity.

Pharmacometabonomics studies have been used to predict the degree of liver damage caused by acetaminophen, the adverse reactions of antitumor drugs, and the central neurotoxicity caused by isoniazid (15-18). Backshall et al. measured the metabolic profile data of 54 patients with colorectal cancer by using H NMR spectroscopy (19). They found that the serum metabolome data before the administration could accurately distinguish patients with grade 0 and grade 3 adverse reactions after administration. Furthermore, they found that a variety of low-density lipoproteins and other compounds in serum were positively correlated with toxicity and could predict the degree of toxicity. However, no metabonomics study predicting the toxicity of AZA has yet been conducted. In this study, we investigate the plasma metabolic type prior to drug administration, aiming to predict AZA-induced myelotoxicity. Several endogenous compounds were identified as biomarkers to predict AZA-induced myelotoxicity in patients with rheumatological disease from Fujian Province, China.

\section{EXPERIMENTAL}

\section{Drugs and reagents}

AZA (50 mg) was obtained from Excella $\mathrm{GmbH}$ Pharmaceutical Co., Ltd. Hydroxychloroquine (0.2 g) was obtained from SPH Zhongxi Pharmaceutical Co., Ltd. HPLC-grade acetonitrile, formic acid, deionized water, and methanol were obtained from Merck (Germany).

\section{Patients}

We recruited 52 patients with SLE. All of the patients visited Quanzhou First Hospital Affiliated with Fujian Medical University, Fujian, China between January 2018 and November 2019. Inclusion criteria included (1) diagnosis of rheumatological disease according to the criteria of the American College of Rheumatology; and (2) informed patient consent. Exclusion criteria included (1) hematologic disease or abnormal hematologic parameters (WBC count $<3.5^{*} 10^{9} / \mathrm{L}$, PLT count $<1.50 * 10^{9} / \mathrm{L}$ ), (2) severe hepatic failure and/or renal insufficiency, (3) malignancy, (4) pregnancy, and (5) medication that may interact with AZA or affect its metabolism. The study was approved by the Ethics Committee of Quanzhou First Hospital Affiliated with Fujian Medical University, the number of the approved ethics committee is [2016] 124, and written informed consent was received from the patients prior to examination.

\section{Study design}

Patients who fulfilled the inclusion criteria were recruited. The initial dosage of AZA was $50 \mathrm{mg}$ daily based on the guidelines of the American College of Rheumatology. Patients treated with AZA were administered blood tests weekly in the first month and then once a month thereafter. Evaluation of hematological toxicity was performed according to the Common Terminology Criteria for Adverse Events (CTCAE) Version 4.0. In the treatment of patients, in the event of myelotoxicity, the dose of AZA should be reduced by $50 \%$, or the drug should be discontinued. If cytopenia develops following a $50 \%$ reduction in dose, AZA should be discontinued permanently in most cases. Thirty patients were divided into two groups: (1) an ADR group (patients with myelotoxicity) and (2) a NO ADR group (patients without myelotoxicity). Figure 1 presents a flow diagram of this pilot clinical trial.

\section{Sample preparation}

Plasma samples had been stored at $-80^{\circ} \mathrm{C}$ on dry ice during transportation. Prior to the analysis, frozen plasma was defrosted and suspended at $4^{\circ} \mathrm{C}$. To precipitate proteins, the plasma $(100 \mu \mathrm{L})$ was mixed with $400 \mu \mathrm{L}$ of acetonitrile/methanol $(1: 1 \mathrm{v} / \mathrm{v})$ in a $1.5 \mathrm{~mL}$ tube. After being vortexed for $5 \mathrm{~min}$ and left to stand for $1 \mathrm{~h}$ at $-20^{\circ} \mathrm{C}$, the mixture was centrifuged at $4000 \times \mathrm{g}$ for $15 \mathrm{~min}$. We transferred the supernatant to a new Eppendorf tube and then evaporated it to dry in a Speed Vac concentrator. Finally, the residues were resuspended in $100 \mu \mathrm{L}$ mobile phase for LC/MS analysis. 


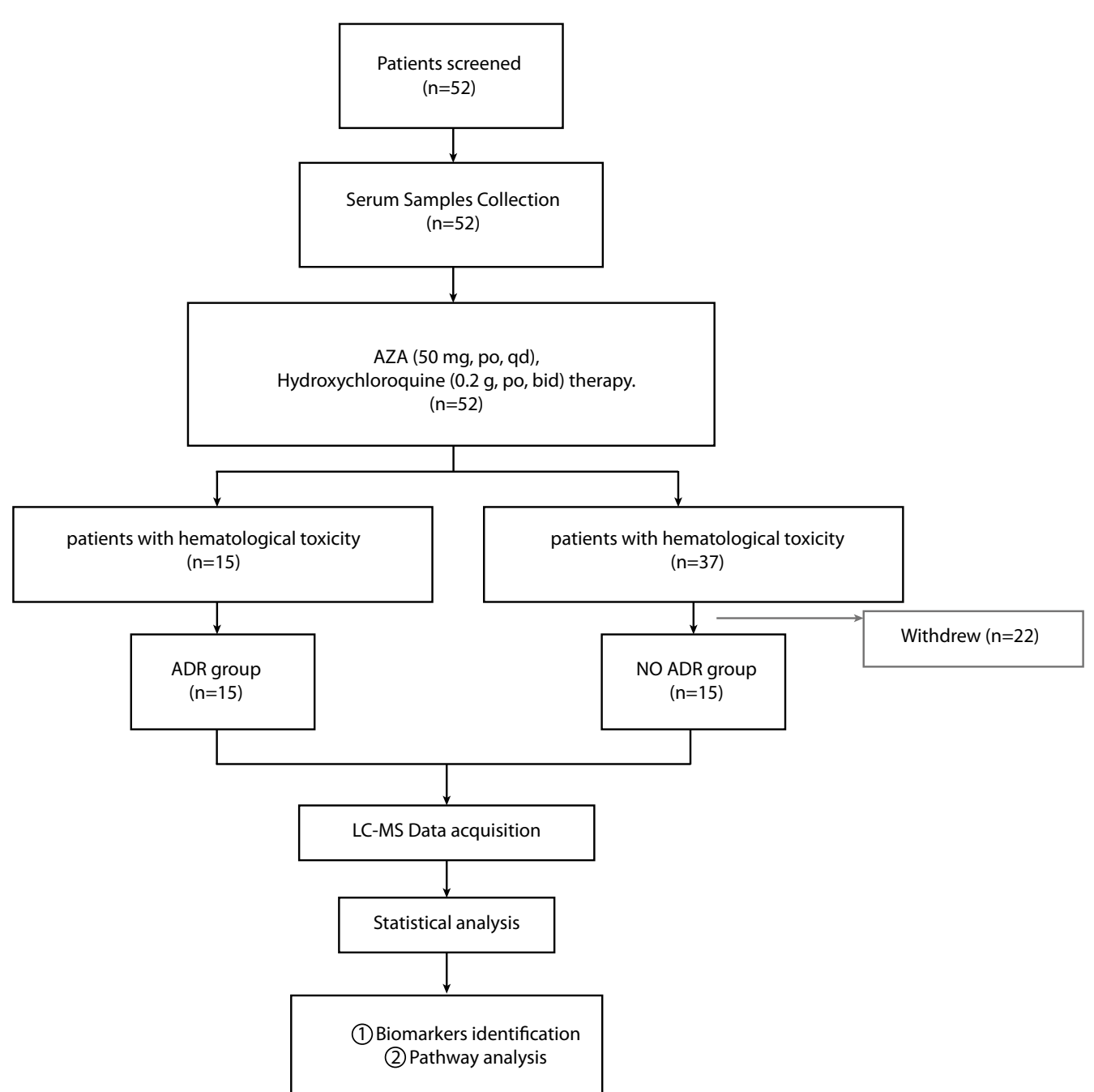

Figure 1. Flow chart of study design.

\section{LC/MS analysis}

UPLC Q-TOF/MS analysis was performed using the Nexera X2 system (Shimadzu Co., Japan) together with a Triple TOF 5600 quadrupole-timeof-flight mass spectrometer (AB SCIEX, USA) as previously described. In brief, metabolites were separated using a ZORBAX Eclipse Plus C18 column $(2.1 \times 100 \mathrm{~mm}$ id, $3.5 \mu \mathrm{m}$, Agilent, USA $)$ maintained at $35^{\circ} \mathrm{C}$. The volume of the sample injection was $3 \mu \mathrm{L}$ for each run conducted in full loop injection mode, and the flow rate of the mobile phase was set as $0.5 \mathrm{~mL} / \mathrm{min}$. In RPLC mode, gradient elution was performed with the solvent system as follows: (A) $0.1 \%$ formic acid and water with (B) $0.1 \%$ formic acid in acetonitrile. The gradient program for mobile phase $\mathrm{A}$ was as follows: $0 \mathrm{~min}, 98 \% \mathrm{~A} ; 13 \mathrm{~min}, 10 \% \mathrm{~A} ; 16 \mathrm{~min}, 10 \% \mathrm{~A}$; $16.1 \mathrm{~min}, 98 \% \mathrm{~A}$ and $20 \mathrm{~min}, 98 \%$. Mass spectrometric analysis was performed with a Triple TOF $5600+$ instrument with an ESI source. The data in positive- and negative-V-geometric modes were acquired for LC/MS analysis. The MS parameters are shown in Table 1.

\section{Metabolites identification}

The UPLC Q-TOF/MS data were preprocessed by Marker View software, including retention time alignment, peak discrimination, filtering,

Table 1. The MS parameters.

\begin{tabular}{|c|c|}
\hline Parameter & Value \\
\hline Capillary voltage & $3000-4500 \mathrm{~V}$ \\
\hline Cone gas & $50 \mathrm{~L} / \mathrm{h}$ \\
\hline Desolvation gas & $600 \mathrm{~L} / \mathrm{h}$ \\
\hline Source temperature & $120^{\circ} \mathrm{C}$ \\
\hline Desolvation temperature & $500^{\circ} \mathrm{C}$ \\
\hline Scan range & $50-1500 \mathrm{~m} / \mathrm{z}$ \\
\hline
\end{tabular}


alignment, matching, and identification. Retention time (tR), m/z value, and peak intensity were obtained. Feature annotations for the reverse liquid phase chromatography streams were performed by matching MS1 and MS2 spectral matching against in Human Metabolome Database (http://www.hmdb. ca/) Metabocard, PubChem description, and KEGG pathways.

\section{Statistical analysis}

The baseline characteristics of patients were compared between the two groups by the $\chi 2$ or Student's t-test. The Mann-Whitney U test was used to perform other comparisons between the ADR and NO ADR groups. Multivariate analysis was conducted by using the Metabo Analyst 4.0 program. Principal component analysis (PCA) and partial least-squares discriminant analysis (PLSDA) models were constructed to identify differential metabolites between the two groups. The metabolite ions were determined according to $\mathrm{m} / \mathrm{z}$, and the Human Metabolome Database (HMDB) (http://www.hmdb.ca/) was used for screening the potential biomarkers (20). The molecular weight tolerance was set at $\pm 0.05 \mathrm{Da}$. The area under the ROC curve was calculated for each potential biomarker. Differences were considered significant at a $\mathrm{P}$ value less than 0.05 . Enrichment analysis and pathway analysis were performed by Metabo Analyst 4.0. SPSS version 22 was used for all other analyses.

\section{RESULTS}

\section{Clinical characteristics}

We recruited 52 patients who were diagnosed with SLE; all of them received AZA (50 mg, po, qd) therapy combined with hydroxychloroquine $(0.2 \mathrm{~g}$, po, bid) therapy. Adverse event-related mortality was not observed during the study period. Fifteen patients with myelotoxicity and fifteen patients without myelotoxicity were assigned to the ADR group and NO ADR group, respectively, while twenty-two patients without myelotoxicity withdrew (Fig.1). Myelotoxicity included agranulocytosis, leukopenia, and thrombocytopenia. After taking AZA, agranulocytosis occurred within one month, ranging from 21 to 28 days. The appearance time of leukopenia and Thrombocytopenia in most cases were included 3 months after treatment. Only one patient developed leukopenia in 7 months after treatment. 15 patients with myelotoxicity were cured after drug withdrawal.

The patients' baseline characteristics are summarized in Table 2. There were no significant differences in sex, age, body weight, albumin, hemoglobin, lymphocyte, TPMT, or NUDT15 between the two groups.

\section{Metabolomic profiles of the ADR and NO ADR groups}

Metabolic profiles of the two groups were acquired by UPLC Q-TOF/MS analysis. To distinguish

Table 2. Baseline demographics.

\begin{tabular}{|c|c|c|c|}
\hline Variable & NO ADR group & ADR group & P-value \\
\hline $\mathrm{n}$ & 15 & 15 & 0.705 \\
\hline Sex $(\mathrm{M} / \mathrm{F})$ & $5 / 10$ & $6 / 9$ & 0.677 \\
\hline Age $($ years $)$ & $42.07 \pm 11.65$ & $40.33 \pm 10.87$ & 0.688 \\
\hline Body weight $(\mathrm{kg})$ & $52.33 \pm 5.61$ & $53.13 \pm 5.19$ & 0.095 \\
\hline Albumin $(\mathrm{g} / \mathrm{L})$ & $34.06 \pm 5.08$ & $30.99 \pm 4.64$ & 0.728 \\
\hline Hemoglobin $(\mathrm{g} / \mathrm{L})$ & $103.47 \pm 25.14$ & $106.47 \pm 21.57$ & 0.068 \\
\hline Lymphocyte $\left(10^{*} / \mathrm{L}\right)$ & $2.24 \pm 0.46$ & $2.53 \pm 0.36$ & 0.309 \\
\hline TPMT & & & 14 \\
\hline Wild type & 15 & 1 & 0.232 \\
\hline Mutant type & 0 & 9 & \\
\hline NUDT15 & & 6 & \\
\hline Wild type & 3 & & \\
\hline Mutant type & 12 & & \\
\hline
\end{tabular}

$* \mathrm{P}<0.05$ and $\uparrow \mathrm{P}<0.01$, ADR vs. NO ADR groups

RA: rheumatoid arthritis; SLE: systemic lupus erythematosus; TPMT: Thiopurine S-methyltransferase; NUDT15: nucleoside diphosphate-linked moiety X-type motif 15. 

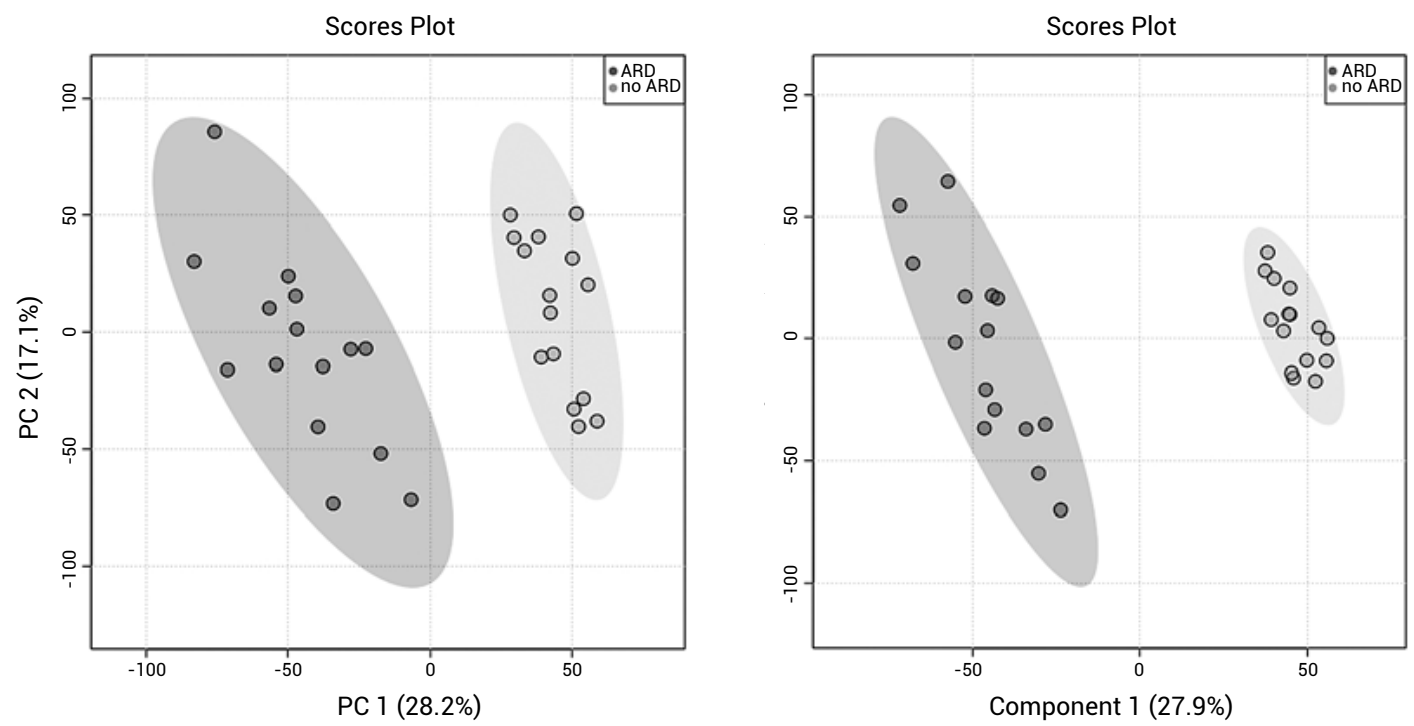

Figure 2. Score plot of the PCA model (A) and PLS-DA models (B) for samples collected from two isolates of sample data.

the ADR group from the NO ADR group by their UPLC Q-TOF/MS spectra and examined the differences between the groups in endogenous metabolism, PCA was performed. The ADR group and NO ADR group each contained 15 samples. The score plot of the PCA model is shown in Figure 2A and reveals different clustering of metabolites between the two groups. We performed PLS-DA to compare the metabolic differences between the two groups and further improve their separation. The PLS-DA model score plot (Figure 2B) indicated a significant separation between the ADR group and the NO ADR group.

After screening in the HMDB database, 1775 different metabolites were identified according to our criteria of $\mathrm{FC}>2$ or $\mathrm{FC}<0.5$ and $\mathrm{p}<0.05$. As shown in Figure 3, these metabolites predominantly included metabolites Lipids and lipid-like molecules $(67.25 \%)$, Organic acids and derivatives $(9.02 \%)$, Organoheterocyclic compounds (5.52\%), Benzenoids $(5.15 \%)$, and Organic oxygen compounds $(4.51 \%)$, followed by other markers.

\section{Model analysis}

PCA and PLS-DA models were constructed to further investigate the metabolic differences between the two groups. $\mathrm{R}^{2}$ was used to evaluate the validity of each model, and $\mathrm{Q}^{2}$ was used to describe its prediction ability. $\mathrm{R}^{2}$ and $\mathrm{Q}^{2}$ were 0.9996 and 0.9786 , respectively, for the PLS-DA model. The results showed that the PLS-DA model has great applicability, being able to distinguish the ADR group from the NO ADR group.

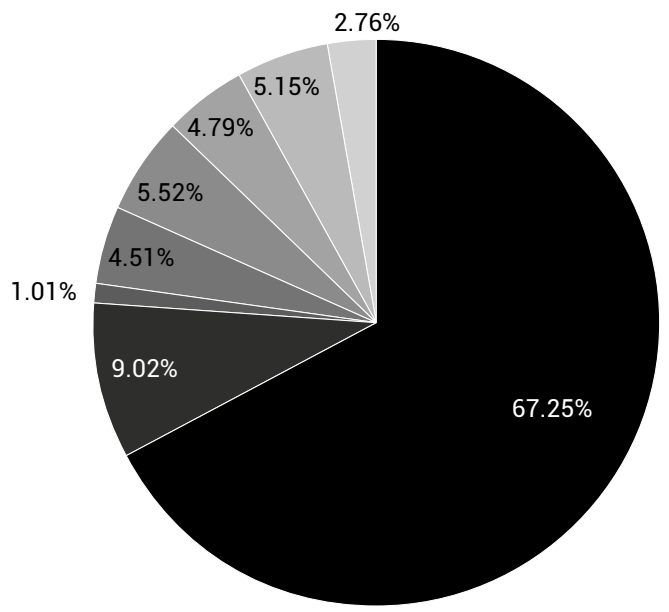

Lipids and lipid-like molecules

Organic acids and derivatives

Organic nitrogen compounds

Organic oxygen compounds

Organoheterocyclic compounds

Phenylpropanoids and polyketides

Benzenoids

others

Figure 3. Pie chart of differential metabolomics. 


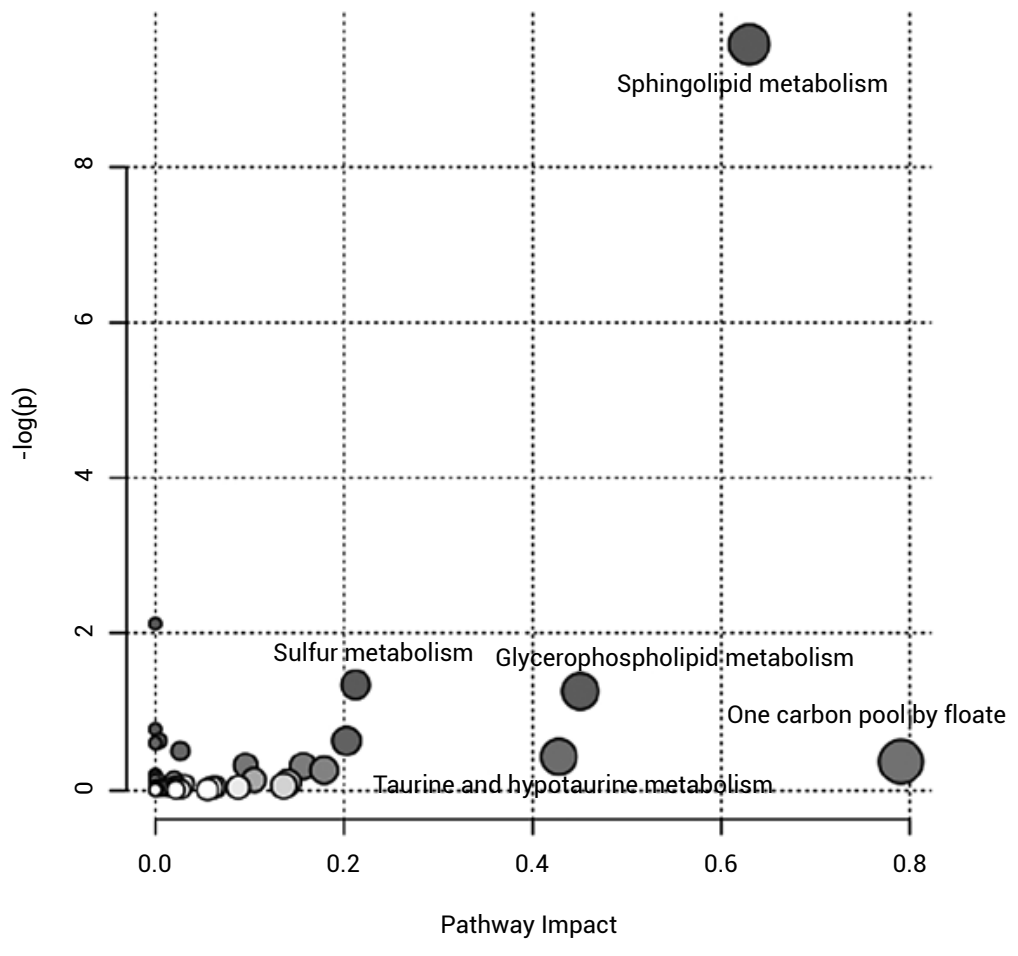

Figure 4. Pathway enrichment analysis. The size and color of each circle were based on pathway impact value and $p$-value, respectively.

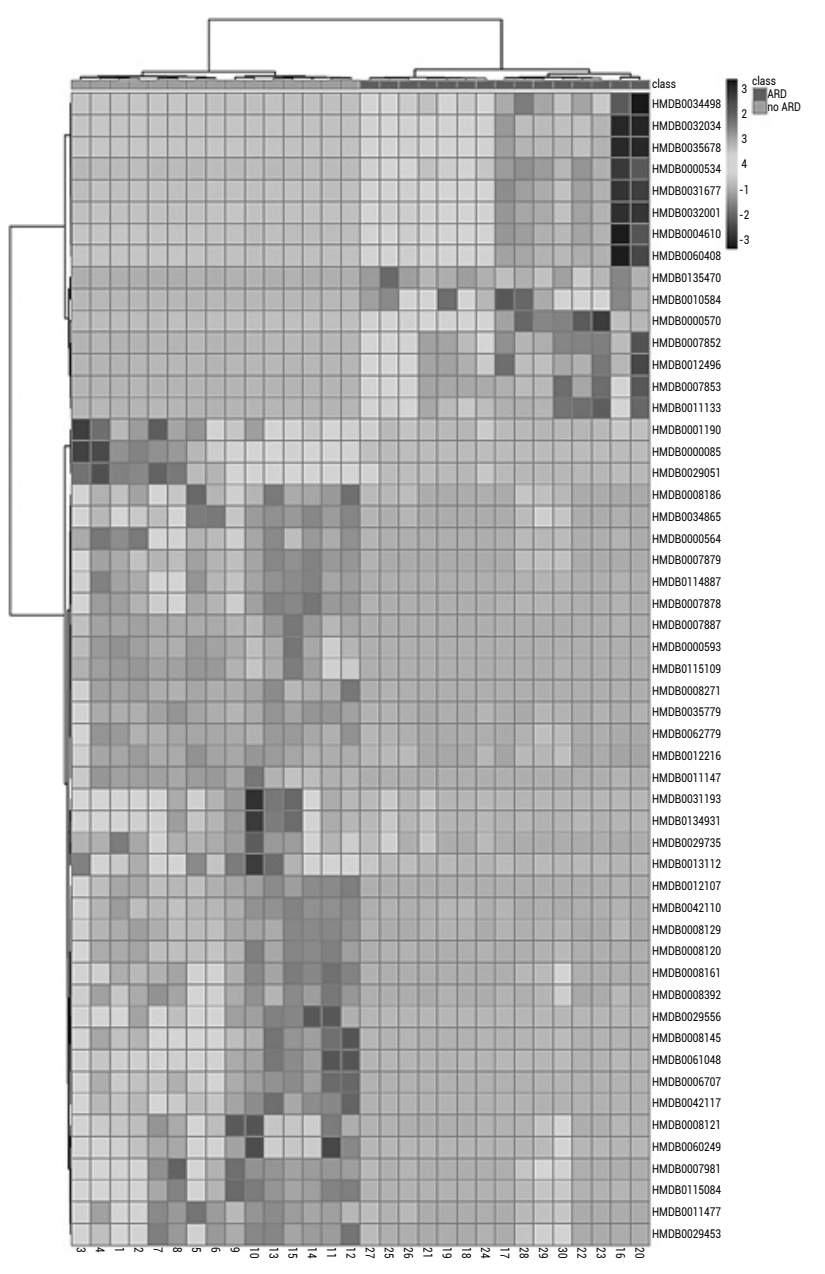

\section{Pathway analysis}

KEGG pathway analysis revealed the involvement of these 1775 metabolites in 40 KEGG pathways. The most significantly enriched pathway $(\mathrm{P}=6.99 \mathrm{E}-05)$, with an impact factor of 0.63 (Figure 4), was Sphingolipid metabolism, with 10 metabolites identified in this pathway.

\section{Biomarkers for ADR diagnosis}

To improve the screening of potential biomarkers to distinguish the ADR group from the NO ADR group, stricter criteria were set: $\mathrm{P}<10 \mathrm{E}-05, \mathrm{FC}>10$ or $\mathrm{FC}<0.1$ and VIP $>1$. The candidate biomarkers and possible metabolite pathways are shown in Table 3. Twenty-two compounds met the above criteria and were identified as potential biomarkers of the ADR group. The corresponding heatmap is shown in Figure 5. The levels of diacylglycerol (DG(18:0e/2:0/0:0)), Dimethylarsinous acid, phosphatidylcholine (PC(14:0/22:1(13Z), PC(18:1(9Z)/18:1(9Z), PC(20:0/18:3(6Z,9Z,12Z), PC(18:1(9Z)/P18:1(9Z)), PC(14:0/20:1(11Z)), PC(18:1(9Z) /22:4(7Z,10Z,13Z,16Z)), PC(14:0/20:0), PC(16:0/16:0), PC(18:3(6Z,9Z,12Z)/22: 


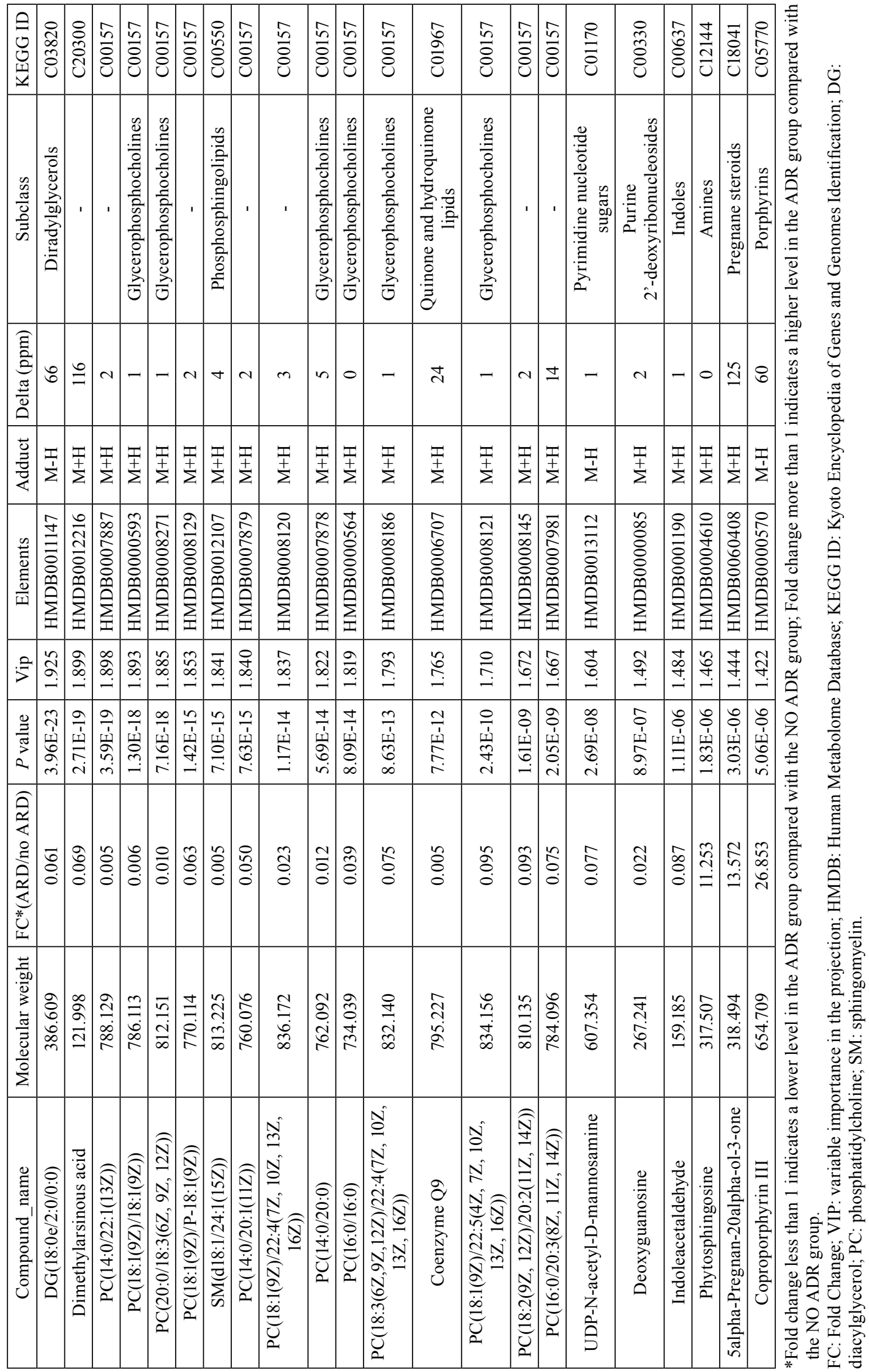




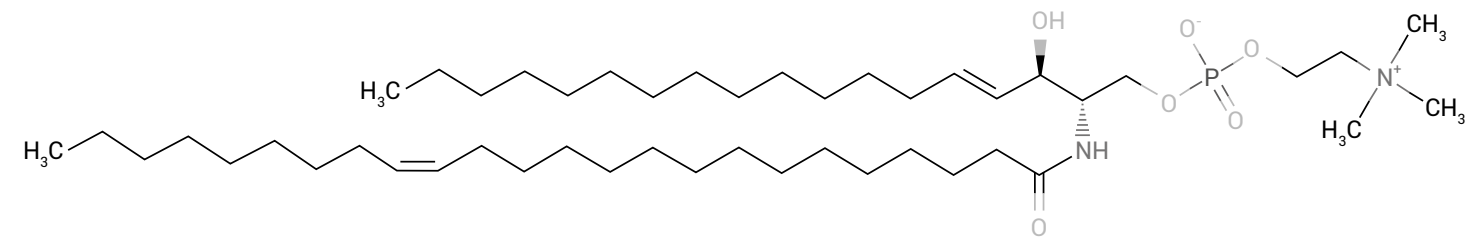

Figure 6 . The chromatograms and chemical 2D structure of sphingomyelin.<smiles>CCCCCCCCCCCCCC[C@@H](O)[C@H](O)[C@H](N)CO</smiles>

Figure 7. The chromatograms and chemical 2D structure of phytosphingosine.

4(7Z,10Z,13Z,16Z)), PC(18:1(9Z)/22:5(4Z,7Z,10 Z,13Z,16Z)0), PC(18:2(9Z,12Z)/20:2(11Z,14Z)), PC(16:0/20:3(8Z,11Z,14Z))), sphingomyelin (SM(d18:1/24:1(15Z)), Coenzyme Q9, UDP-Nacetyl-D-mannosamine, Deoxyguanosine and Indoleacetaldehyde in the ADR group were significantly lower than the corresponding levels in the NO ADR group, whereas those of phytosphingosine, 5alpha-Pregnan-20alpha-ol-3-one, Coproporphyrin III were significantly higher in the ADR group than those in the NO ADR group. Two of the above 22 metabolites, which were identified as involved in Sphingolipid metabolism, were selected as candidate biomarkers for ADR, namely, sphingomyelin SM(d18:1/24:1(15Z)) and phytosphingosine. Their chromatograms and chemical two-dimensional structures are shown in Figure 6 and Figure 7. The $P$ values associated with biomarkers sphingomyelin and phytosphingosine were $7.10 \mathrm{E}-15$ and $1.83 \mathrm{E}-06$, respectively, and the AUCs were 1.0 and 1.0, respectively (Figure 8).

A

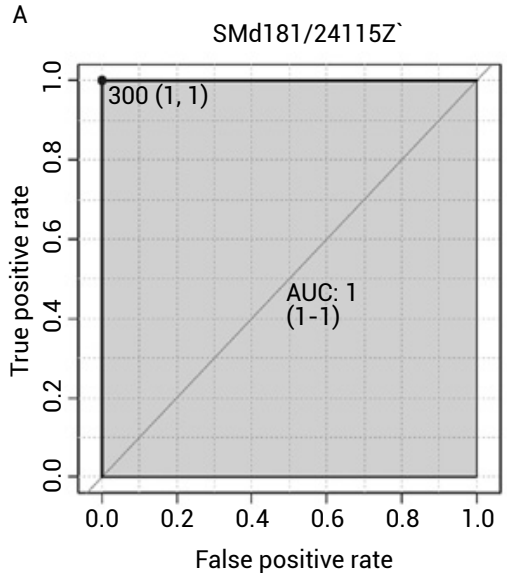

\section{DISCUSSION}

The incidence of the rheumatological disease is increasing yearly. Although biological agents, which are expensive, have a good therapeutic effect, immunosuppressants, such as AZA, continue to play an important role in treatment. AZA is a prodrug that is converted to 6-MP via glutathione transferase (GST) (21). There are three metabolic pathways for the further metabolism of 6-MP. In one pathway, 6-MP is converted to 6-thiouric acid (6-TU), which is an inactive metabolite, by xanthine oxidase (XO). XO is mainly distributed in the intestine; it also occurs in the liver. In the second pathway, it is converted to 6-thio-hypoxanthine monophosphate (6-TIMP) by hypoxanthine phosphoribosyltransferase (HGPRT). Then, 6-TIMP is converted into 6-thioguanine monophosphate (6-TGMP) under the action of inosine monophosphate dehydrogenase (IMPDH) and guanine monophosphate synthase (GMPS), and 6-TGMP converts to 6-thioguanine triphosphate (6-TGTP)

B

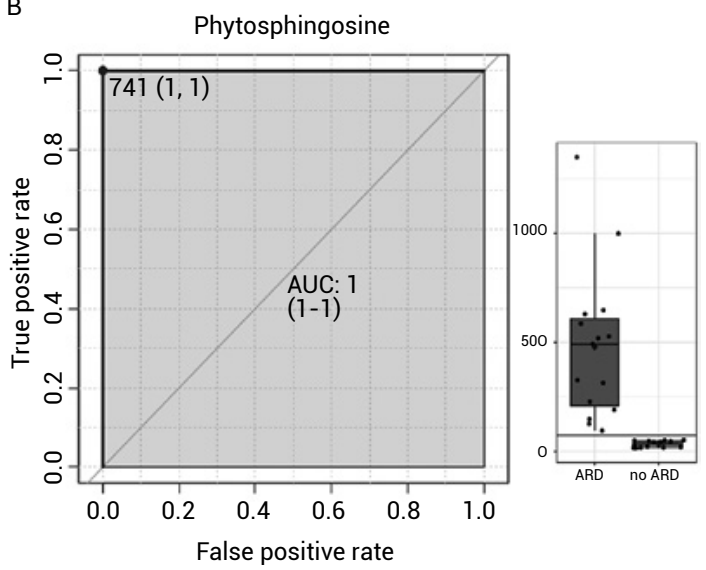

Figure 8. Receiver operating characteristic (ROC) curve analysis. A The P-value and AUC for sphingomyelin; B The P-value and AUC for phytosphingosine. 
and 2-deoxy-6-thioguanine triphosphate (2-deoxy6 -TGTP) in a reaction catalyzed by nucleoside diphosphate kinase (NDPK). In the third pathway, 6-MP is converted to 6-methylmercaptopurine (6MeMP), which is an inactive product, by TPMT (22-23). There are many factors affecting the metabolic pathway of AZA, so the incidence of adverse reactions is high. The most prominent adverse reaction of AZA is hematotoxicity. In this study, we discovered that the incidence of AZA-induced myelotoxicity was $29 \%$, which is consistent with the incidence in the Chinese patient population reported in previous studies of $27-41.3 \%$ (24).

Polymorphisms of the TPMT gene lead to enzyme inactivation or significant decreases in enzyme function and increase the risk of treatmentrelated myelotoxicity (25). Nevertheless, TPMT genotyping has not been widely performed because the cost-effectiveness of routine testing and the optimal clinical environment are not yet clear (26). Our previous studies revealed that the frequency of TPMT variants associated with AZA-induced myelotoxicity was low (1\%) (9), which agrees with the results reported by other studies (27). We also found no significant association between TPMT polymorphism and AZA-induced myelotoxicity (9). TPMT polymorphism cannot effectively predict the occurrence of AZA-induced myelotoxicity, suggesting that this toxicity is caused by multiple factors. To predict AZA-induced myelotoxicity, it is necessary to identify biomarkers that predict myelotoxicity after AZA treatment using pretreatment serum samples from patients with rheumatological disease.

This is the first study to report that sphingomyelin and phytosphingosine are associated with AZA-induced myelotoxicity, and these metabolites might represent new biomarkers. In our study, lower serum levels of sphingomyelin and higher serum levels of phytosphingosine were detected in patients who suffered myelotoxicity than in patients without myelotoxicity. Some studies have reported that high levels of sphingomyelin, achieved via SMS1 overexpression, basic fibroblast growth factor-dependent stimulation of SMS, or exogenous sphingomyelin, promote cell growth (28). Our results showed that the level of sphingomyelin in the ADR group was significantly lower than that in the NO ADR group. Previous studies have suggested that a high level of sphingosine promotes cell death; high levels can occur as a result of SK inhibition, exogenous sphingosine treatment, or, in cancer cells, induced by environmental stress, chemotherapeutic treatment, or apoptotic stimulus (28). There are many studies on SK inhibitors, which inhibit the proliferation of cancer cells. SKI-II is reported to be a nonselective inhibitor of SKs and to induce cell apoptosis (29). M. Pitman et al. found that MP-A08, which inhibits SK1 and SK2, can suppress proliferation in many cancer cell lines (30). Sphingosine inducing apoptosis is the product of ceramide hydrolysis by ceramidase $(31,32)$. The structure of phytosphingosine is similar to that of sphingosine. phytosphingosine is thought to combat tumor cells by affecting one or more nutrient transport systems, and phytosphingosine analogs are used as anticancer drugs $(33,34)$. We found increased levels of phytosphingosine in rheumatic patients with AZA-induced myelotoxicity in our research. Therefore, we hypothesized that sphingomyelin and phytosphingosine may serve as potential biomarkers for AZA-induced myelotoxicity of rheumatic patients. There are no reports about the relationships between the levels of sphingomyelin and phytosphingosine and AZA-induced myelotoxicity; investigations of these associations are needed.

The two candidate biomarkers identified in this study are involved in one metabolic pathway, which is sphingolipid metabolism. Sphingolipids are important parts of eukaryotic cells and are lipids with various structures, comprising more than 4000 different species (35). Sphingolipid metabolism can be imagined as a massively complex network composed of various sphingolipid molecules and enzymes involved in regulating cell growth, differentiation, autophagy, senescence, cell death, and signal transduction (36-39). It is reported that ceramide, dihydroceramide, GluCer, LacCer, SM, and S1P are associated with atherosclerotic plaques and that sphingolipid metabolism may be a new therapeutic target (40). Jacob $\mathrm{M}$ et al (41). found that plasma ceramide levels were increased in patients with type 2 diabetes. In addition, studies suggest that serum levels of aSMase can predict mortality in patients at risk of systemic inflammation (42). Some studies have also shown that targeting sphingolipid metabolism may be of use in cancer therapy. Investigators have found that $\mathrm{C} 16$ ceramide can increase cell sensitivity to chemotherapy drugs and promote apoptosis (43). Sphingolipid metabolism is reported to be important in cisplatin-induced apoptosis and drug resistance (44). These observations suggest that sphingolipids affect organismal biology. Therefore, we assume that sphingolipid metabolism is associated with AZA-induced myelotoxicity. Studies should be performed to elucidate the mechanism of sphingolipid metabolism in rheumatic patients with AZA-induced myelotoxicity. 
We chose UPLC/MS-based metabolomic profiling for identifying metabolic biomarkers in rheumatic patients with AZA-induced myelotoxicity. This study has the following limitations: First, the sample size was small; larger cohorts of rheumatic patients should be enrolled in future research. Second, only one dose of AZA-induced myelotoxicity was considered. Future studies should compare the effects of different doses of AZA on metabolic results. Candidate biomarkers and the mechanisms require further investigation to validate our findings.

\section{CONCLUSION}

In conclusion, several metabolic characteristics differed significantly between patients with and without myelotoxicity. phingomyelin and phytosphingosine were reported for the first time to be metabolite biomarkers for predicting AZA-induced myelotoxicity. Further investigations are required to validate the findings and provide references for individualized treatments for patients with rheumatological disease.

\section{Acknowledgments}

This research was supported by Grants from the Natural Science Foundation of Fujian Province, China (No. 2017J01351 to Y-FL), and Fujian Medical University Sailing Fund, China (No. 2016QH086 to Y-FL).

\section{Conflict of interest}

The authors declare no conflicts of interest.

\section{REFERENCES}

1. Gabriel S.E., Michaud K.: Arthritis Res. Ther. 11, 229 (2009).

2. Garcia S.: Int. J. Mol. Sci. 20, 374 (2019).

3. Payne K., Newman W., Fargher E., Tricker K., Bruce IN., Ollier W.E.: Rheumatology (Oxford) 46, 727 (2007).

4. Gastal G.R., Moreira S., Noble C.F., Ferreira L.E., França P.H., Pinho M.: Arq. Gastroenterol. 49, 130 (2012).

5. Patel A.N., Langan S.M., Batchelor J.M.: Br. J. Dermatol. 166, 701 (2012).

6. Weinshilboum R.: Drug Metab. Dispos. 29, 601 (2001).

7. Lichtenstein G.R., Abreu M.T., Cohen R., Tremaine W., American Gastroenterological
Association: Gastroenterology 130, 940 (2006).

8. Jun J.B., Cho D.Y., Kang C., Bae S.C.: Clin. Exp. Rheumatol. 23, 873 (2005).

9. Su S.S., Lin Y.F., Zhou H.: Chin. Med. J. (Engl.) 133, 1002 (2020).

10. Nicholson J.K., Lindon J.C.: Nature 455, 1054 (2008).

11. Lindon J.C., Nicholson J.K., Holmes E.: The Handbook of Metabonomics and Metabolomics. Elsevier, Amsterdam, 2007.

12. Everett J.R.: Handb. Exp. Pharmacol. 2019, 263 (2019).

13. Zhao Y.Y., Liu J., Cheng X.L., Bai X., Lin R.C.: Clin. Chim. Acta 413, 642 (2012).

14. Dunn W.B.: The Handbook of Metabolic Phenotyping. The Role of Ultra Performance Liquid Chromatography-Mass Spectrometry in Metabolic Phenotyping. 97 (2019).

15. Kwon H.N., Kim M., Wen H., Kang S., Yang H.J., et al.: Kidney Int. 79, 529 (2011).

16. Cunningham K., Claus S.P., Lindon J.C., Holmes E., Everett J.R., et al.: J. Proteome Res. 11, 4630 (2012).

17. Clayton T.A., Baker D., Lindon J.C., Everett J.R., Nicholson J.K.: Proc. Natl. Acad. Sci. USA 106, 14728 (2009).

18. Winnike J.H., Li Z., Wright F.A., Macdonald J.M., O’Connell T.M., Watkins P.B.: Clin. Pharmacol. Ther. 88, 45 (2010).

19. Backshall A., Sharma R., Clarke S.J., Keun H.C.: Clin. Cancer Res. 17, 3019 (2011).

20. Wishart D.S., Knox C., Guo A.C., Eisner R., Young N., et al.: Nucleic Acids Res. 37, 603 (2009).

21. Lennard L., Van Loon J.A., Weinshilboum R.M.: Clin. Pharmacol. Ther. 46, 149 (1989).

22. Bourgine J., Garat A., Allorge D., CrunelleThibaut A., Lo-Guidice J.M., et al.: Pharmacogenet. Genomics 21, 313 (2011).

23. Poppe D., Tiede I., Fritz G., Becker C., Bartsch B., et al.: J. Immunol. 176, 640 (2006).

24. Fei X., Shu Q., Hua B.Z., et al.: Medicine (Baltimore). 97 (2018).

25. Zelinkova Z., Derijks L.J., Stokkers P.C., Vogels E.W., van Kampen A.H., et al.: Clin. Gastroenterol. Hepatol. 4, 44 (2006).

26. Donnan J.R., Ungar W.J., Mathews M., Hancock-Howard R.L., Rahman P.: Pediatr. Blood Cancer. 57, 231 (2011).

27. Kakuta Y., Naito T., Onodera M., Kuroha M., Kimura T., et al.: Pharmacogenomics J. 16, 280 (2016). 
28. Hannun Y.A., Obeid L.M.: Nat. Rev. Mol. Cell Biol. 19, 175 (2018).

29. Gustin D.J., Li Y., Brown M.L., Min X., Schmitt M.J., et al.: Bioorg. Med. Chem. Lett. 23, 4608 (2013).

30. Pitman M.R., Powell J.A., Coolen C., Moretti P.A., Zebol J.R., et al.: Oncotarget 6, 7065 (2015).

31. Cuvillier O., Edsall L., Spiegel S.: J Biol Chem. 275, 15691 (2000).

32. Coroneos E., Wang Y., Panuska J.R., Templeton D.J., Kester M.: Biochem. J. 316, 13 (1996).

33. Garsi J.B., Sernissi L., Vece V., Hanessian S., McCracken A.N., et al.: Eur. J. Med. Chem. 159, 217 (2018).

34. Nagahara Y., Shinomiya T., Kuroda S., Kaneko N., Nishio R., Ikekita M.: Cancer Sci. 96, 83 (2005).

35. Sud M., Fahy E., Cotter D., Brown A., Dennis E.A., et al.: Nucleic Acids Res. 35, 527 (2007).
36. Ryland L.K., Fox T.E., Liu X., Loughran T.P., Kester M.: Cancer Biol. Ther. 11, 138 (2011).

37. Tirodkar T.S., Voelkel-Johnson C.: Exp. Oncol. 34, 231 (2012).

38. Hannun Y.A., Obeid L.M.: Nat. Rev. Mol. Cell Biol. 9, 139 (2008).

39. Klionsky D.J.: Nat. Rev. Mol. Cell Biol. 8, 931 (2007).

40. Edsfeldt A., Dunér P., Stahlman M., Mollet I.G, Asciutto G, et al.: Arterioscler. Thromb. Vasc. Biol. 36, 1132 (2016).

41. Haus J.M., Kashyap S.R., Kasumov T., Zhang R., Kelly K.R., et al.: Diabetes 58, 337 (2009).

42. Kott M., Elke G., Reinicke M., Winoto-Morbach S., Schädler D., et al.: PLoS One 9, e112323 (2014).

43. Truman J.P., García-Barros M., Obeid L.M., Hannun Y.A.: Biochim. Biophys. Acta 1841, 1174 (2014).

44. Li Y.L., Lin M.L., He S.Q., Jin J.F.: World J. Transl. Med.. 5, 37 (2016). 